\title{
Performance Analysis of A Multi-channel MAC with Dynamic CCH Interval in WAVE System
}

\author{
Dongbi Zhu ${ }^{1, a}$ and Dandan Zhu ${ }^{1, b}$ \\ ${ }^{1}$ Department of Electronics and Communication Engineering, \\ College of Engineering, Yanbian University, Yanji, China \\ adbzhu@ybu.edu.cn, bzdd0513@ybu.edu.cn
}

Keywords: WAVE system; Multi-channel MAC; $\mathrm{CCH}$ interval; saturation throughput

\begin{abstract}
To improve the throughput performance of the Wireless Access in Vehicular Environment (WAVE) system, we propose a multi-channel MAC protocol that is able to adaptively adjust the intervals of Control Channel $(\mathrm{CCH})$ and Service Channel $(\mathrm{SCH})$ according to the probability distribution of the reservation time for service packet in $\mathrm{CCH}$ Interval. Numerical results show that our protocol can significantly improve the performance of WAVE system.
\end{abstract}

\section{Introduction}

WAVE system is a radio communications system intended to provide seamless, inter- operable services to transportation. IEEE802.11p [1] defines a single channel MAC for WAVE vehicles. Recently, the IEEE1609.4 [2] is considered as a default multi-channel MAC standard for WAVE system, which defines the general framework for multi-channel management.

This WAVE MAC adopts the split phrase mode [3] to coordinate multiple channels. Channel access time is divided into Synchronization Intervals (Sync Interval) with a length of 100ms, consisting of a CCH Interval and a SCH Interval, 50ms of each. During CCH Interval, all the devices have to monitor $\mathrm{CCH}$, where safety frame, vehicle status frame, and WAVE service announcement (WSA), are delivered. When SCH Interval arrives, devices can optionally switch to SCHs, which are used for non-safety applications [4].

In a congested vehicular traffic condition, the limited length of $\mathrm{CCH}$ is unable to provide sufficient bandwidth to deliver a large amount of safety packets and control packets. On the other hand, if the vehicle density is sparse, the occasional transmission on the $\mathrm{CCH}$ channel will waste some of $\mathrm{CCH}$ interval, so that some large bandwidth consuming applications cannot obtain enough bandwidth resource on the SCHs. Hence the fixed length of CCH interval of WAVE MAC has certain limitations in improving the performance of WAVE system. This is confirmed by data in [5]. Q. Wang et al. [6] proposed a Variable CCH Interval (VCI) MAC protocol which can optimize the intervals based on the average time of reservation for service packet in $\mathrm{CCH}$ interval.

In this paper we propose a dynamic $\mathrm{CCH}$ interval for vehicle environment, named Dynamic $\mathrm{CCH}$ Interval (DCI) MAC protocol in WAVE system, which is able to adaptively adjust the intervals of $\mathrm{CCH}$ and $\mathrm{SCH}$ according to the probability distribution of the reservation time for service packet in $\mathrm{CCH}$ Interval. An algorithm for our protocol is employed to calculate the duration of optimal $\mathrm{CCH}$ interval based on the traffic condition.

\section{The Proposed Protocol}

Multi-Channel Coordination Mechanism. We use a coordination mechanism that provides contention-free $\mathrm{SCH}$ s by the channel reservation on $\mathrm{CCH}$. As show in Fig. 1, $\mathrm{CCH}$ interval is divided into Safety Interval and WSA Interval. A new $\mathrm{CCH}$ interval begins from the Safety Interval, during which vehicles transmit safety information. It is known that $T_{s a}$ is proportional to the number of vehicles in WAVE system and inverse proportion to the transmission data rate of $\mathrm{CCH}$ [6]. 
During the $\mathrm{CCH}$ Interval, service providers broadcast WSA packets, piggyback with the identity of the offered service and SCH ID. Other nodes that need the service can respond with an acknowledgment (ACK). Then, a positive ACK will indicate the access interval and the SCH ID to be used. After the $\mathrm{CCH}$ interval, nodes optionally switch to SCHs and transmit service data. The duration of $\mathrm{CCH}$ interval should be optimized to achieve the best case, i.e., the number of reservations made on $\mathrm{CCH}$ equals the number of packets transmitted on all $\mathrm{SCHs}$.

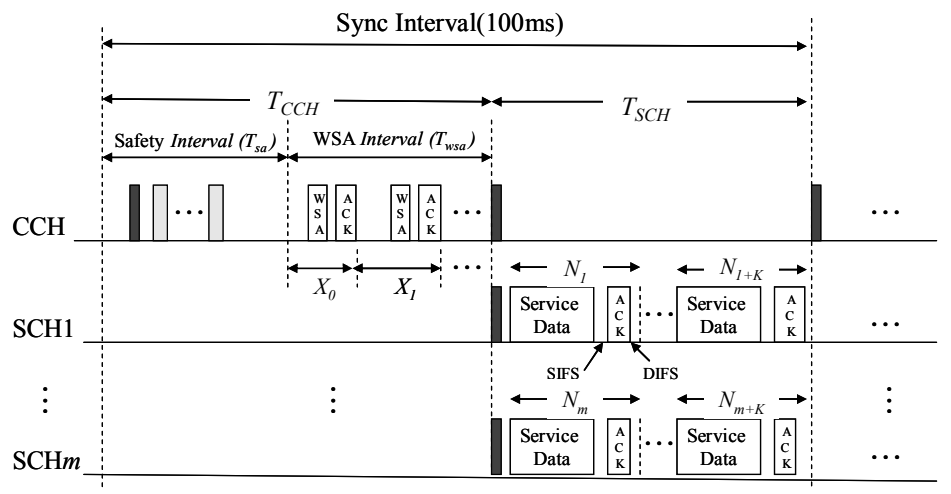

Fig.1 The analytical model

Algorithm to calculate the duration of CCH Interval. Let $T_{C C H}$ and $T_{S C H}$ represent the length of $\mathrm{CCH}$ interval and SCH interval, respectively, and $T_{\text {data }}$ be the fixed length of data packet transmitted on SCH. $K$ is the maximum number of data packet which can transmitted on SCH interval, it determines the length of $T_{S C H}$. We define $Y$ as the time interval that since WAVE vehicles start to contend $\mathrm{CCH}$ until $m \times K$ th reservation of WSA frame is made successfully and $F_{Y}(z)$ be its probability generating function. The optimal value of $K^{*}$ and $T_{C C H}^{*}$ can be obtained by the algorithm as shown Fig.2. In the algorithm $\varepsilon$ is the predetermined performance threshold.

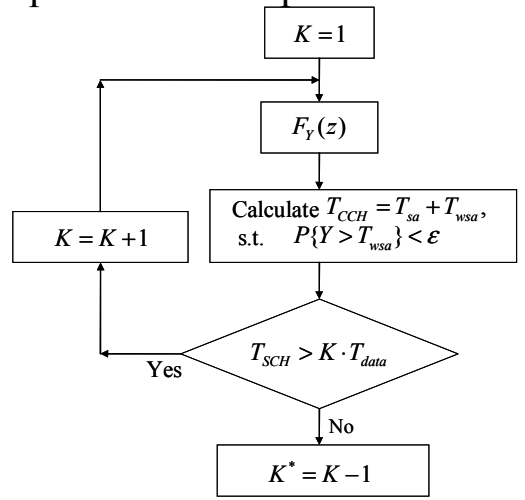

Fig.2 The algorithm of our MAC protocol

Considering a heavy traffic situation, each vehicle always has a WSA packet to send in every synchronization interval, and at least one vehicle intends to respond. Let $n$ be the total number of WAVE vehicles and $m$ be the number of SCH channels in the WAVE system. Let $\tau$ be the probability that a vehicle transmits a packet in arbitrary slot time. The value of $\tau$ can be easily obtained by using the Markov model [7]. Let $P_{I}, P_{S}$ and $P_{C}$ be the probability that no vehicles transmit, only one vehicle transmit more than one vehicle transmits in a given slot, respectively.

$$
P_{I}=(1-\tau)^{n}, \quad P_{S}=n \tau(1-\tau)^{n-1}, \quad P_{C}=1-P_{I}-P_{S} .
$$

We define $X_{0}$ as the time interval from the beginning of the WSA Interval to the ending of successful transmission of the first WSA/RFS packet and $X_{i}(i \geq 1)$ as the time interval from the ending of successful transmission of the $i^{\text {th }}$ WSA packet to the ending of successful transmission of the $(i+1)^{\text {th }}$ WSA packet as see in Fig.3. It is obvious that $\left\{X_{n} \mid n \geq 0\right\}$ are i.i.d random variables. We can now express $F(z)$ that the probability generating function of $X_{0}$, i.e., $F(z)=\sum_{k=0}^{\infty} P\left\{x_{0}=k\right\} z^{k}$. 
First, note that

$$
\left\{\begin{array}{l}
P\left(x_{0}=k\right)=0, \quad \text { if } 0 \leq k \leq j \\
P\left(x_{0}=k\right)=P_{I}^{k-j} P_{S}, \quad \text { if } j \leq k \leq 2 j \\
P\left(x_{0}=k\right)=P_{I} P\left\{x_{0}=k-1\right\}+P_{C}\left(x_{0}=k-j\right), \quad \text { if } k \geq 2 j
\end{array}\right.
$$

According to $Y=X_{0}+X_{1}+\ldots+X_{M}(M=m \times K)$, we have

$$
F_{Y}(z)=(F(z))^{M}=\left[\frac{P_{S} z^{j}}{1-P_{I} z-P_{C} z^{j}}\right]^{m K} .
$$

Under the condition $P\left\{Y>T_{C C H}<\varepsilon\right\}$, we obtain that

$$
T_{C C H}>j m K+\log _{P_{I}}\left(\frac{\left(1-P_{I}\right)^{m K}}{P_{S}^{m K}} \cdot \varepsilon\right) .
$$

We can now calculate the minimum $T_{C C H}$ according to (4) while ensuring the value of $K$, and finally obtain the optimal $T_{C C H}$ by the algorithm of Fig. 2 .

\section{Performance Evaluation}

To evaluate our protocol, we compare the current WAVE MAC and the VCI MAC with our DCI MAC. The experimental parameters refer to the default IEEE 802.11p [8].

CCH Interval Duration. Fig. 3 show the optimal intervals obtained by theoretical calculation under different number of vehicles. In a congested network environment, DCI MAC protocol can provide longer $\mathrm{CCH}$ interval to ensure the reliable transmission of safety information. On the other hand, if the vehicle density is sparse, VCI MAC protocol can improve the channel utilization by increasing the length of SCH interval.

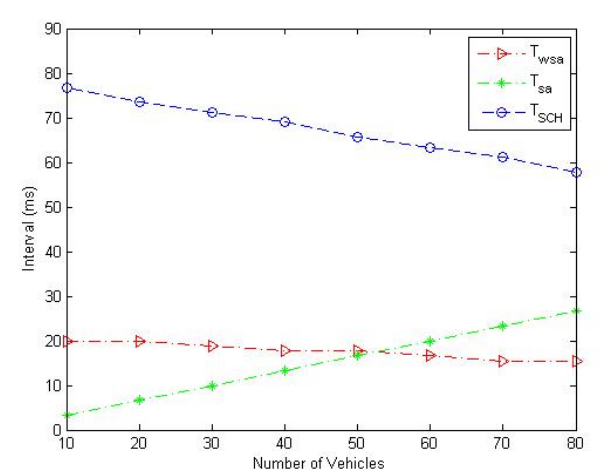

Fig.3 Optimum intervals under different number of vehicles

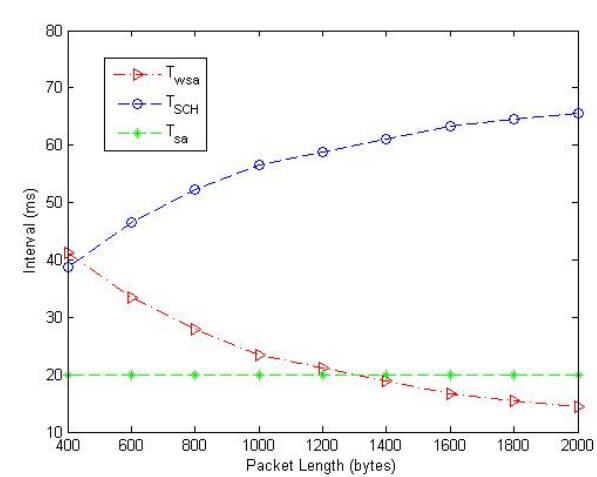

Fig.4 Optimal intervals under different packet lengths.

Fig. 4 depicts the optimal intervals under different packet lengths. It's reasonable that WSA interval reduces correspondingly while the service packet length increases, and the length of safety interval keeps unchanged according to the fixed value of $n$.

Saturation Throughput on SCHs. As show in Fig.5, the throughput of WAVE MAC almost maintains regardless of the increase of $n$ because of the fixed length of SCH interval, while our DCI MAC enjoys higher throughput than both of the others MAC under all different number of vehicles. 


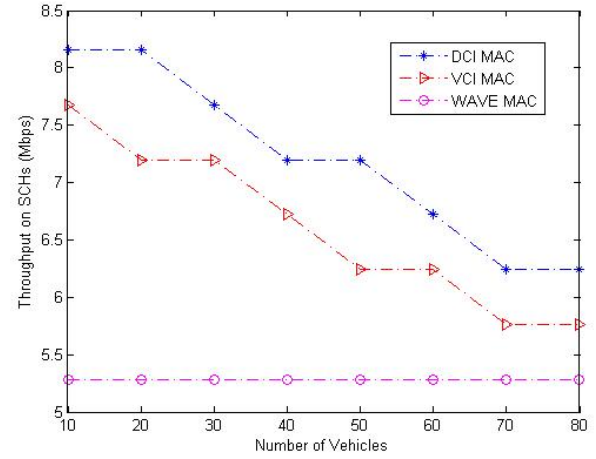

Fig.5 Saturation throughput comparison under different number of vehicles

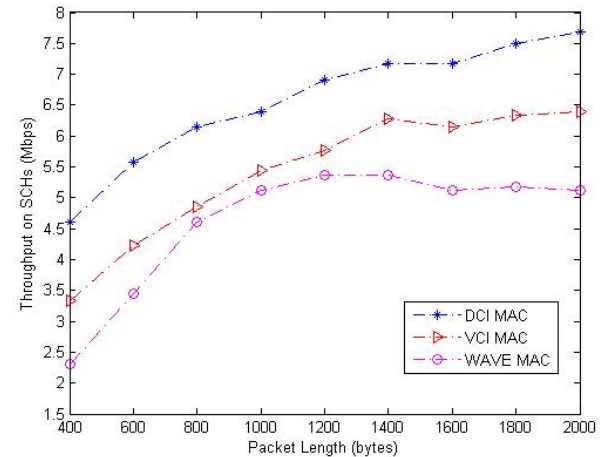

Fig.6 Saturation throughput comparison under different packet length

The reason why the throughput reduces rapidly when the number of vehicle increases is that with more vehicles accessing the WAVE system, the network become more and more crowded. At this moment, it's necessary to reserve enough time of safety interval to ensure the reliable transmission of safety information.

Fig. 6 shows the saturation throughput under different service packet length, and it indicates that the proposed DCI MAC scheme is able to improve the saturation throughput of SCHs significantly, especially when the service packet length is long.

\section{Conclusion}

In this paper, we propose a multi-channel MAC protocol with dynamic interval division, named DCI MAC protocol, for WAVE system. We use a coordination mechanism that provides contention-free $\mathrm{SCH}$ by the channel reservation on $\mathrm{CCH}$. An algorithm is proposed to calculate the duration of $\mathrm{CCH}$ interval according to the probability distribution of the reservation time for service packet in $\mathrm{CCH}$ Interval. The numerical results show that our protocol improves the throughput performance significantly under different network traffic condition.

\section{References}

[1] IEEE Standards 802.11p, Amendment6: Wireless Access in Vehicular Environments, 2010.

[2] IEEE Standards 1609.4, IEEE Trial-Use Standard for Wireless Access in Vehicular Environments (WAVE) - Multi-channel Operation, 2006.

[3] J. Mo, H. S. W. So and J. Walrand, Comparison of Multichannel MAC Protocols, IEEE Transactions on Mobile Computing, vol. 7, no. 1, 2008:50-65.

[4] L. Liu, W. W. Xia, L. F. Shen, An Adaptive Multi-channel MAC Protocol with Dynamic Interval Division in Vehicular Environment, The 1st International Conference on Information Science and Engineering. Nanjing, 2009: 2534-2537.

[5] S. Y. Wang, H. L. Chao, K.C. Liu et al., Evaluating and Improving the TCP/UDP Performance of IEEE 802.11(p)/1609.4 Network. ISCC 2008: 163-68.

[6] Q. Wang, P. L. Su, H. R. Fu, et al., An Enhanced Multi-channel MAC for the IEEE 1609.4 based Vehicular Ad Hoc Networks, IEEE INFOCOM 2010 proceeding. San Diego, USA, 2010:1-2.

[7] G. Bianchi, Performance Analysis of the IEEE 802.11 Distributed Coordination unction, IEEE journal on selected areas in communication, 2000, 3(18): 535-547.

[8] IEEE P802.11p/D3.0, Draft Amendment for Wireless Access in Vehicular Environment (WAVE), July, 2007. 